\title{
Anomalous high-magnetic field electronic state of the nematic superconductors $\mathrm{FeSe}_{1-x} \mathrm{~S}_{x}$
}

\author{
M. Bristow $\odot,{ }^{1}$ P. Reiss $\odot,{ }^{1}$ A. A. Haghighirad,${ }^{1,2}$ Z. Zajicek $\odot,{ }^{1}$ S. J. Singh, ${ }^{1}$ T. Wolf, ${ }^{2}$ D. Graf, ${ }^{3}$ W. Knafo, ${ }^{4}$ \\ A. McCollam, ${ }^{5}$ and A. I. Coldea ${ }^{1}{ }^{1, *}$ \\ ${ }^{1}$ Clarendon Laboratory, Department of Physics, University of Oxford, Parks Road, Oxford OX1 3PU, United Kingdom \\ ${ }^{2}$ Institute for Quantum Materials and Technologies (IQMT), Karlsruhe Institute of Technology, 76021 Karlsruhe, Germany \\ ${ }^{3}$ National High Magnetic Field Laboratory and Department of Physics, Florida State University, Tallahassee, Florida 32306, USA \\ ${ }^{4}$ Laboratoire National des Champs Magnétiques Intenses (LNCMI-EMFL), UPR 3228, CNRS-UJF-UPS-INSA, 143 Avenue de Rangueil, \\ 31400 Toulouse, France \\ ${ }^{5}$ High Field Magnet Laboratory (HFML-EMFL), Radboud University, 6525 ED Nijmegen, The Netherlands
}

(Received 20 September 2019; revised manuscript received 18 December 2019; accepted 9 January 2020; published 13 March 2020)

\begin{abstract}
Understanding superconductivity requires detailed knowledge of the normal electronic state from which it emerges. A nematic electronic state that breaks the rotational symmetry of the lattice can potentially promote unique scattering relevant for superconductivity. Here, we investigate the normal transport of superconducting $\mathrm{FeSe}_{1-x} \mathrm{~S}_{x}$ across a nematic phase transition using high-magnetic fields up to $69 \mathrm{~T}$ to establish the temperature and field dependencies. We find that the nematic state is dominated by a linear resistivity at low temperatures that evolves towards Fermi-liquid behavior, depending on the composition $x$ and the impurity level. Near the nematic end point, we find an extended temperature regime with $\sim T^{1.5}$ resistivity, different from the behavior found near an antiferromagnetic critical point. The variation of the resistivity exponent with temperature reflects the importance of the nematoelastic coupling that can also suppress divergent critical fluctuations at the nematic end point. The transverse magnetoresistance inside the nematic phase has a $\sim H^{1.55}$ dependence over a large magnetic field range and it displays an unusual peak at low temperatures inside the nematic phase. Our study reveals anomalous transport inside the nematic phase, influenced by both changes in the electronic structure and the scattering with the lattice and spin fluctuations.
\end{abstract}

DOI: 10.1103/PhysRevResearch.2.013309

Magnetic field is a unique tuning parameter that can suppress superconductivity to reveal the normal low-temperature electronic behavior of many unconventional superconductors $[1,2]$. High-magnetic fields can also induce new phases of matter, probe Fermi surfaces, and determine the quasiparticle masses from quantum oscillations in the proximity of quantum critical points $[1,3]$. In unconventional superconductors, close to antiferromagnetic critical regions, an unusual scaling between a linear resistivity in temperature and magnetic fields was found [4,5]. Magnetic fields can also induce metal-toinsulator transitions, as in hole-doped cuprates, where superconductivity emerges from an exotic electronic ground state [2].

FeSe is a unique bulk superconductor with $T_{c} \sim 9 \mathrm{~K}$, which displays a variety of complex and competing electronic phases [6]. FeSe is a bad metal at room temperature and it enters a nematic electronic state below $T_{s} \sim 87 \mathrm{~K}$. This nematic phase is characterized by multiband shifts driven by orbital ordering that lead to Fermi-surface distortions [6,7]. Furthermore, the

\footnotetext{
*Corresponding author: amalia.coldea@physics.ox.ac.uk

Published by the American Physical Society under the terms of the Creative Commons Attribution 4.0 International license. Further distribution of this work must maintain attribution to the author(s) and the published article's title, journal citation, and DOI.
}

electronic ground state is that of a strongly correlated system and the quasiparticle masses display orbital-dependent enhancements $[7,8]$. FeSe shows no long-range magnetic order at ambient pressure, but complex magnetic fluctuations are present at high energies over a large temperature range [9]. Below $T_{s}$, the spin-lattice relaxation rate from nuclear magnetic resonance (NMR) experiments is enhanced as it captures the low-energy tail of the stripe spin fluctuations $[10,11]$. Furthermore, recent $\mu \mathrm{SR}$ studies invoke the close proximity of FeSe to a magnetic quantum critical point as the muon relaxation rate shows unusual temperature dependence inside the nematic state [12].

The changes in the electronic structure and magnetic fluctuations of FeSe can have a profound implication on its transport and superconducting properties. Scanning tunneling microscopy (STM) reveals a highly anisotropic superconducting gap driven by orbital-selective Cooper pairing [13]. Due to the the presence of the small inner bands, whose Fermi energies are comparable to the superconducting gap, FeSe was placed inside the BCS-BEC crossover regime [14]. In large magnetic fields, when the Zeeman energy is comparable to the gap and Fermi energies, a peculiar, highly polarized superconducting state may occur [14].

To establish the role played by different competing interactions on nematicity and superconductivity, an ideal route is provided by the isoelectronic substitution of selenium by sulfur ions in $\mathrm{FeSe}_{1-x} \mathrm{~S}_{x}$ [15]. This tuning parameter suppresses 
nematicity and leads to changes in the electronic structure, similar to the temperature effects, with the Fermi surface becoming isotropic in the tetragonal phase and the electronic correlations becoming weaker $[3,6,15,16]$. As nematicity is suppressed, it creates ideal conditions to explore a potential nematic critical point [17] in the absence of magnetism. The superconducting dome extends outside the nematic state, but anisotropic pairing remains robust [18], and a different superconducting state was suggested to be stabilized in the tetragonal phase [19].

In this paper, we study the normal electronic state across the nematic transition in $\mathrm{FeSe}_{1-x} \mathrm{~S}_{x}$ using magnetotransport studies in high-magnetic fields up to $69 \mathrm{~T}$. We find that the nematic state shows unusual transport behavior with temperature and transverse magnetoresistance $\left(\sim H^{1.55}\right)$, reflecting an unconventional scattering mechanism. Just outside the nematic phase, resistivity is dominated by a $\sim T^{1.5}$ dependence, similar to studies under pressure [20]. The transverse magnetoresistance is significant inside the nematic phase and it shows an unusual change in slope at low temperatures. Inside the nematic phase at low temperatures, we find linear resistivity followed by Fermi-liquid behavior for certain $x$ and impurity levels. We identify a qualitative link between spin fluctuations and the linear resistivity regime. The variation of the resistivity exponent with temperature suggests that the nematoelastic coupling plays an important role in these systems [21,22].

Methods. Single crystals of $\mathrm{FeSe}_{1-x} \mathrm{~S}_{x}$ were grown by the $\mathrm{KCl} / \mathrm{AlCl}_{3}$ chemical vapor transport method [23]. The composition for samples from the same batch was checked using energy-dispersive $\mathrm{x}$-ray (EDX) spectroscopy, as reported previously in Ref. [3]. Note that in Refs. [24,25], the nominal $x_{\text {nom }}$ is used to identify the composition (which is the value used during the growth process), which typically corresponds to about $80 \%$ of the real $x$ (see also Refs. [3,17,26]). The structural transition at $T_{S}$ also provides useful information about the expected $x$ value, as shown in Fig. $\mathrm{S} 1$ in the Supplemental Material (SM) [27]. More than 30 samples were screened for high-magnetic field studies to test their physical properties. The residual resistivity ratio varies between 15 and 44 , and we observed the variation within the same batch due to the inhomogeneous distribution of sulfur with increasing $x$, as shown in Figs. S1c and S8 in the SM [27]. We estimate that the nematic end point is located close to $x \sim 0.180(5)$ (see Figs. S1 and S11 in the SM [27]).

In-plane transport measurements $[I \|(a b)]$ were performed in a variable-temperature cryostat in dc fields up to $38 \mathrm{~T}$ at HFML, Nijmegen and up to $70 \mathrm{~T}$ at LNCMI, Toulouse, with the magnetic field applied mainly along the $c$ axis (transverse magnetoresistance) but also in the $(a b)$ conducting plane (longitudinal magnetoresistance) at constant temperatures. Low-field measurements were performed in a $16 \mathrm{~T}$ Quantum Design PPMS. The resistivity $\rho_{x x}$ and Hall $\rho_{x y}$ components were measured using a low-frequency five-probe technique (between 15 and $30 \mathrm{~Hz}$ to avoid crosstalk between samples) and were separated by (anti)symmetrizing data measured in positive and negative magnetic fields. Good electrical contacts were achieved by In soldering along the long edge of the single crystals and electrical currents up to $3 \mathrm{~mA}$ were used to avoid heating. Magnetic fields along the $c$ axis suppress superconductivity in fields higher than $20 \mathrm{~T}$ for all $x$ values [3].

Results and discussion. Figures 1(a)-1(e) show the transverse magnetoresistance $\rho_{x x}$ of different single crystals of $\mathrm{Fe}\left(\mathrm{Se}_{1-x} \mathrm{~S}_{x}\right)$ up to $35 \mathrm{~T}$ at various fixed temperatures inside the nematic phase and up to $69 \mathrm{~T}$ for $x \sim 0.25$ in the tetragonal phase. From these constant temperature runs, we can extract the magnetoresistance at fixed fields for each composition $x$, as shown in Figs. 1(f)-1(j), which reveals several striking features. First, the magnetoresistance increases significantly once a system enters the nematic state at $T_{s}$, and its magnitude depends on the concentration $x$, being largest for FeSe, just above $T_{c}$. Second, in the vicinity of $T_{c}$ in magnetic fields much larger than the upper critical field, the magnetoresistance shows an unusual temperature dependence that varies strongly with $x$ across the phase diagram, as shown in Figs. 1(f) and $1(\mathrm{~g})$. The resistivity slope $d \rho_{x x} / d T$ in $34 \mathrm{~T}$ of FeSe changes sign around a crossover temperature, $T^{*} \sim 14 \mathrm{~K}$, as shown in Fig. 1(f) [also in the color plot of the slope in Fig. 3(d)]. With increasing sulfur substitution from FeSe towards $x \sim$ 0.07 (defined as the nematic A region), the position of $T^{*}$ shifts to a slightly higher temperature of $\sim 20 \mathrm{~K}$, and the peak in magnetoresistance is much smaller than for FeSe. For higher concentrations, approaching the nematic phase boundary ( $x \sim 0.11-0.17$ defined as the nematic $\mathrm{B}$ region), there is a small peak at $T^{*}$ but the negative slope $d \rho_{x x} / d T$ in $34 \mathrm{~T}$ is strongly enhanced at low temperatures, which is different from the nematic A phase [see Figs. 1(h), 1(i) and 3(d)]. Lastly, in the tetragonal phase, the magnetoresistance shows a conventional behavior and increases quadratically in magnetic fields [Figs. 1(e) and 1(j)].

The unusual downturn in resistivity in high-magnetic fields below $T^{*}$ inside the nematic A phase was previously assigned to large superconducting fluctuations in FeSe in magnetic fields up to $16 \mathrm{~T}[10,11]$. We find that this behavior remains robust in magnetic fields that are at least a factor of 2 higher than the upper critical field of $\sim 16 \mathrm{~T}$ for $H \| c$ [10]. Furthermore, it also manifests in $x \sim 0.07$ inside the nematic A phase, but it disappears for higher $x \gtrsim 0.1$. As $T_{c}$ and the upper critical field inside the nematic phase for different $x$ remain close to that of FeSe $[3,28]$, the changes in the resistivity slope in high magnetic fields are likely driven by field-induced effects that influence scattering and/or the electronic structure.

The Hall coefficient, $R_{H}=\rho_{x y} / \mu_{0} H$, extrapolated in the low-field limit (below $1 \mathrm{~T}$ ) for $\mathrm{FeSe}_{1-x} \mathrm{~S}_{x}$ has an unusual temperature dependence, as shown in Fig. 2(b). For a compensated metal, the sign of the Hall coefficient depends on the difference between the hole and electron mobilities [29]. In the tetragonal phase above $T_{s}$ and for $x \gtrsim 0.18, R_{H}$ is close to zero [Fig. 2(b)], as expected for a two-band compensated metal. On the other hand, in the low-temperature nematic A phase, the sign of $R_{H}$ is negative, suggesting that transport is dominated by a highly mobile electron band $[15,30]$. It becomes positive inside the nematic $\mathrm{B}$ phase, dominated by a holelike band [Fig. 2(a)]. It is worth mentioning that inside the nematic $\mathrm{B}$ phase, the quantum oscillations are dominated by a low-frequency pocket with light mass that disappears at the nematic end point [3]. Thus, the behavior of $R_{H}$ is 
(a)
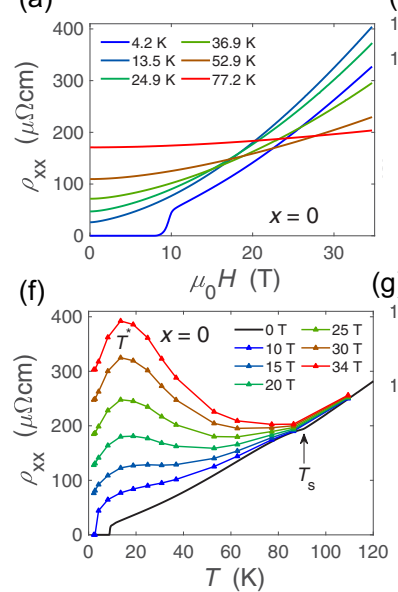

(b)

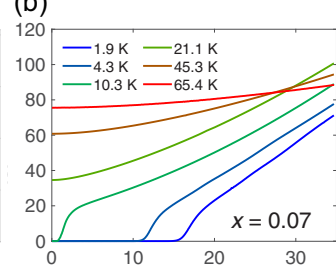

(g) $\quad \mu_{0} H(\mathrm{~T})$

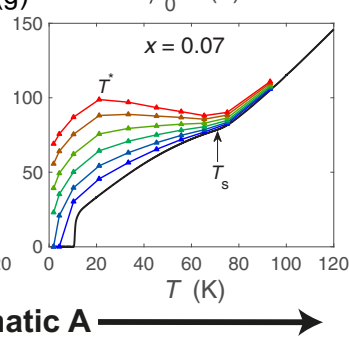

(c)
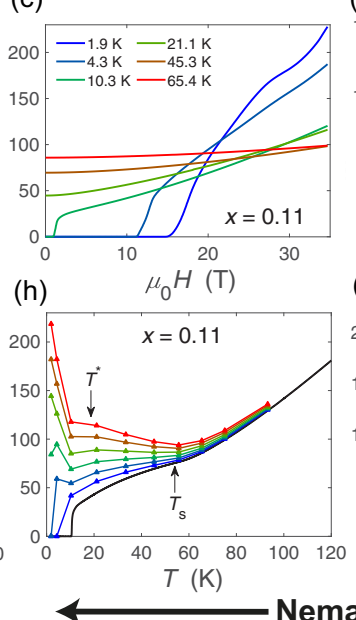

(d)

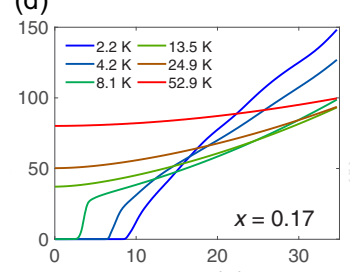

(i) $\quad \mu_{0} H(\mathrm{~T})$
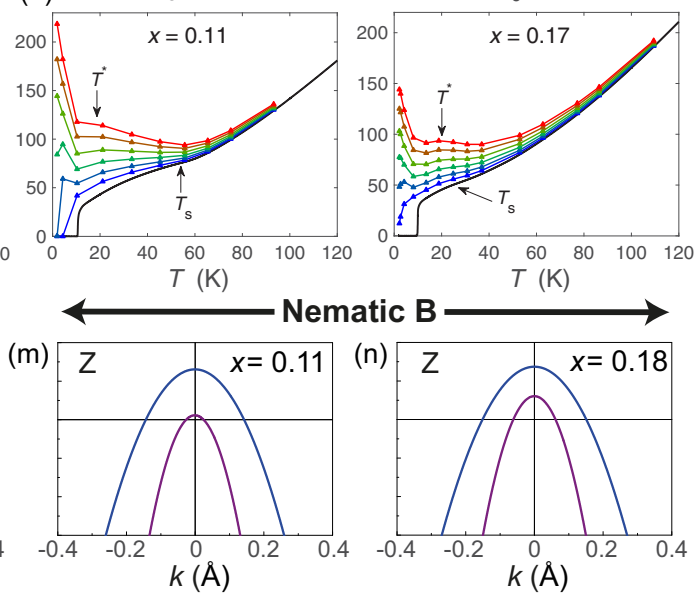

(e)

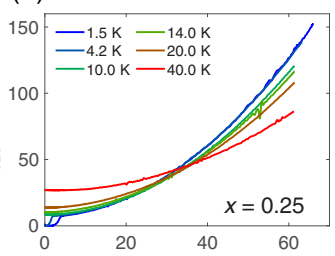

(j) $\quad \mu_{0} H(T)$

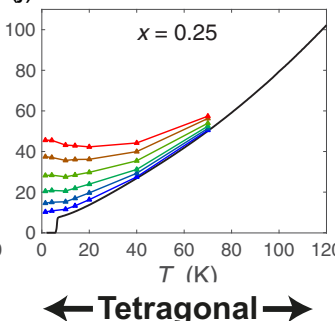

\begin{tabular}{l|l} 
(o) $\mathrm{A}$ & $\mathrm{A}=0.18$ \\
\hline
\end{tabular}

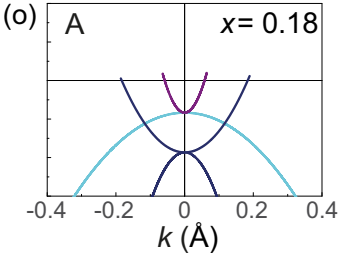

FIG. 1. Transverse magnetoresistance of the nematic and tetragonal $\mathrm{FeSe}_{1-x} \mathrm{~S}_{x}$. (a)-(e) Field-dependent in-plane resistivity at different constant temperatures for different compositions, $x$, inside and outside the nematic phase. The magnetic field is applied along the $c$ axis, perpendicular to the in-plane electrical current. A strong magnetoresistance develops inside the nematic phase. (f)-(j) Resistivity against temperature in zero field (solid line) and at fixed magnetic fields (symbols), as extracted from the top panel for different $x$. The peak in magnetoresistance is indicated by $T^{*}$ and the nematic phase emerges at $T_{s}$. (k)-(o) Schematic band dispersion at low temperatures at two highsymmetry points at the top of the Brillouin zone, $Z$ and $A$, for different $x$ (based on ARPES data reported in Refs. [6,7,15,16]). The horizontal lines represent the location of distinct regions in the magnetotransport behavior called nematic $\mathrm{A}(x=0,0.07)$, nematic $\mathrm{B}(x=0.11,0.17)$, and the tetragonal phase for $x \gtrsim 0.18$. In the tetragonal phase, the compensated semimetal is formed of two electron and two holelike bands. Deep inside the nematic phase, the inner hole band and inner electron bands are brought in the vicinity of the Fermi level.

linked to the disappearance of a small three-dimensional (3D) hole pocket center at the $Z$ point in FeSe below $T_{s}$ and its reemergence in the nematic B phase with $x$ substitution around $x \sim 0.11$, as found in angle-resolved photoemission spectroscopy (ARPES) studies [15] and sketched in Fig. 1(m). Interestingly, the subtle changes in the electronic structure in $\mathrm{FeSe}_{1-x} \mathrm{~S}_{x}$ seem to correlate with the different features observed both in magnetoresistance [Figs. 1(f)-1(i)] and in the Hall coefficient $\left|R_{H}\right|$ that show a maximum near $T^{*}$ [Fig. 2(b)]. In high-magnetic fields, the Hall component of FeSe is complex, changing sign and being nonlinear $[15,28]$. A magnetic field can induce changes in scattering and/or field-induced Fermi-surface effects in the limit when the cyclotron energy is close to the Zeeman energy, in particular in multiband systems that have pockets with small Fermi energies. The smallest inner bands of $\mathrm{FeSe}_{1-x} \mathrm{~S}_{x}$ shift in energy as a function of composition $x$ (and temperature [3]), as shown in Figs. 1(k)-1(o). Furthermore, the Hall effect in iron-based superconductors can be affected by the spin fluctuations that induce mixing of the electron and hole currents [31].

Next, we attempt to quantify the magnetoresistance across the phase diagram and in the vicinity of the nematic end point in $\mathrm{FeSe}_{1-x} \mathrm{~S}_{x}$, as shown in Figs. 1(a)-1(e). At the lowest temperature, inside the nematic phase, the transverse magnetoresistance of most samples is dominated by quantum oscillations [3], making it difficult to quantify its dependence.
A near-linear magnetoresistance is detected for $x \sim 0.07$ in Fig. 1(b) and for a "dirtier" sample with lower residual resistivity ratio of 8.5 (that can result from defect concentrations like dislocations and vacancies) in Fig. S9 in the SM [27]. The quasilinear field magnetoresistance at low temperature can arise from squeezed trajectories of carriers in semiclassically large magnetic fields in the case of small Fermi surfaces $\left(\omega_{c} \tau \gg 1\right)[32,33]$. Another explanation for an almost linear magnetoresistance is the presence of mobility fluctuations caused by spatial inhomogeneities, as found in low carrier density systems [33-35].

Classical magnetoresistance in systems with a single dominant scattering time is expected to follow a $H^{2}$ dependence [32]. This results in Kohler's rule, which is violated in $\mathrm{FeSe}_{1-x} \mathrm{~S}_{x}$, suggesting that the magnetoresistance is not dominated by a single scattering time, as shown in Figs. S2(a)-S2(c) in the SM [27]. Magnetoresistance is quadratic in magnetic fields up to $69 \mathrm{~T}$ in the tetragonal phase $(x \geqslant 0.19)$ (see Fig. 1(e) and Figs. S4(e) and S4(f) in the SM [27]), but not inside the nematic phase. $\mathrm{FeSe}_{1-x} \mathrm{~S}_{x}$ are compensated multiband systems [6] where the high-field magnetoresistance is expected to be very large and dependent on the scattering times of the electron and hole bands [29]. As these systems are very clean and quantum oscillations have been observed, the overall estimated value for the $\omega_{c} \tau$ is 1.4 in $5 \mathrm{~T}$, which places these systems in the high-field limit where the details 

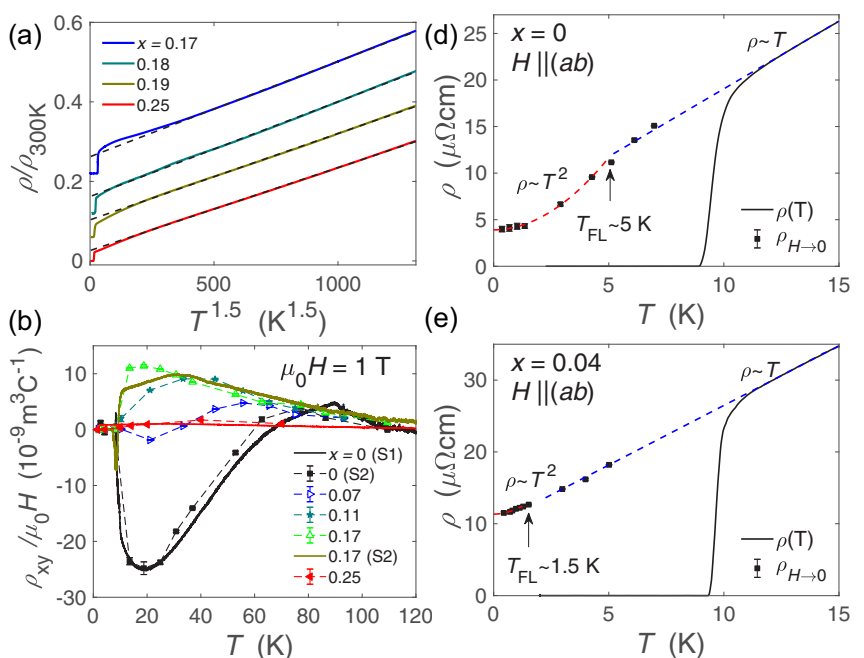

(e)
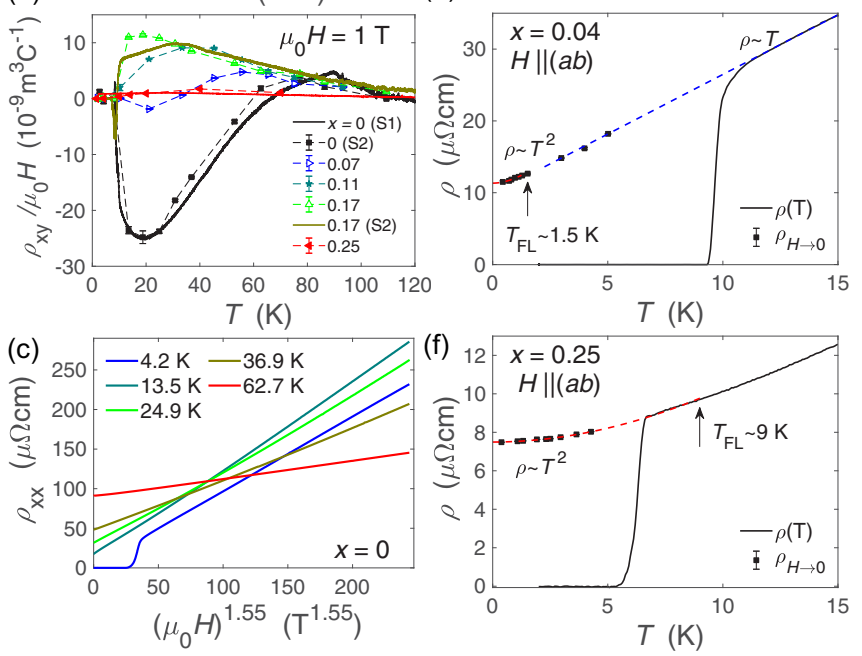

FIG. 2. Normal electronic state of $\mathrm{FeSe}_{1-x} \mathrm{~S}_{x}$. (a) Temperature dependence of resistivity vs $T^{1.5}$ over a large temperature region just outside the nematic phase. Data are shifted vertically for clarity. (b) Hall effect coefficient in low magnetic fields $\left(\mu_{0} H \leqslant 1 \mathrm{~T}\right)$, indicating the change in sign and the dominance of different highly mobile carriers across the nematic phase. (c) Resistivity vs $H^{1.55}$ for FeSe inside the nematic phase at constant temperatures. (d)-(f) The low-temperature linear resistivity. The solid lines are the zero-field resistivity data. Solid circles represent the zero-field extrapolated values of $\rho_{x x}$ when $H \|(a b)$ plane. The dashed lines represent fits to a Fermi-liquid behavior found below $T_{\mathrm{FL}}$, as indicated by arrows.

of the Fermi surface and unusual type of scattering may become important [29,32]. Magnetoresistance has a complex form and instead simpler scaling have been sought to reveal its importance, in particular in the vicinity of critical points [4,5]. For example, in $\mathrm{BaFe}_{2}\left(\mathrm{As}_{1-x} \mathrm{P}_{x}\right)$ for $x \sim 0.33$ at the antiferromagnetic critical point, a universal $H-T$ scaling was empirically found between the linear resistivity in temperature and magnetic field [4]. For $\mathrm{FeSe}_{1-x} \mathrm{~S}_{x}$ near the nematic end point at $x \sim 0.17$, we find that a $H-T$ dependence collapses onto a single curve, as shown in Fig. 2(e) in the SM [27]. Despite this, the energy scaling of magnetoresistance used to described the antiferromagnetic critical point in Ref. [4] is not obeyed in the vicinity of the nematic end point in $\mathrm{FeSe}_{1-x} \mathrm{~S}_{x}$, as detailed in Figs. 2(g)-2(i) in the SM [27]. This could be due to additional constraints to be included either to account for the nematoelastic coupling [21] and/or the effect of additional effects induced by sulphur substitution. For example, a very dirty sample of $\mathrm{FeSe}_{1-x} \mathrm{~S}_{x}$ close to $x_{\text {nom }} \sim 0.18$ was recently suggested to obey $H-T$ scaling [25].

For reasons described above, we propose a different approach to model the magnetoresistance data in the nematic state of $\mathrm{FeSe}_{1-x} \mathrm{~S}_{x}$, using a power law in magnetic fields given by $\rho_{x x}(H)=\rho_{H \rightarrow 0}+b H^{\delta}$ for each temperature.. Strikingly, we find that all the magnetoresistance data inside the nematic phase can be described by a unique exponent $\delta \sim 1.55(5)$ over a large field window, as shown by the color plot in Fig. 3(c) as well as in Figs. 2(c) and Figs. S4(a)- S4(d) in the SM [27]. A detailed method of the extraction of $\delta$ and its stability over a large temperature and field window is shown in Fig. S3 in the SM [27]. Furthermore, this gives $\delta \sim 2$ for samples in the tetragonal phase [see Fig. 3(c)]. Inside the nematic phase, the Fermi surface of $\mathrm{FeSe}_{1-x} \mathrm{~S}_{x}$ distorts anisotropically [6,7] and an unusual type of scattering could become operational due to the presence of hot and cold spots along certain directions [36].

In the absence of magnetic field, the transport behavior can also be described by a power law, $\rho(T)=\rho_{0}+A T^{\gamma}$. Figure 3(a) shows a color plot of the exponent $\gamma$, which is close to unity at low temperatures inside the nematic phase and becomes sublinear close to the nematic phase boundary, indicating a significant deviation from Fermi-liquid behavior (a value of $\gamma=1.1(2)$ was previously reported for FeSe [37]). Outside the nematic phase, a $T^{1.5}$ dependence of resistivity describes the data well over a large temperature range up to $120 \mathrm{~K}$ [see Figs. 2(a) and 3(a)], in agreement with previous studies of $\mathrm{FeSe}_{1-x} \mathrm{~S}_{x}$ under pressure [20]. Using the high-magnetic field data below $T_{c}$, we extract the lowtemperature normal resistivity in the absence of superconductivity, $\rho_{H \rightarrow 0}(T)$. Figures $2(\mathrm{~d})-2(\mathrm{f})$ show resistivity against temperature for different values of $x$, together with the extrapolated high-field points, using longitudinal magnetoresistance when $H \|(a b)$ plane, shown in Figs. S5 and S6 in the SM [27]. We also use transverse magnetoresistance data (in the regime where quantum oscillations were not dominating the response) to extract the zero-field resistivity, using the established power law $H^{1.55}$, as shown in detail in Figs. S4 and S7 in the SM [27]. From both measurements, we find evidence for a linear resistivity in the low-temperature regime, below $T^{*}$, inside the nematic phase. At low temperatures, we observe that Fermi-liquid behavior recovers in the tetragonal phase (see, also, Refs. [24,38]) and inside the nematic phase, below $T_{\mathrm{FL}}$ [see Figs. 2(d)-2(f) and 3(b)]. This is strongly dependent on composition and impurity level, even in the vicinity of the nematic end point (see Figs. S8 and S9 in the SM [27]).

We find that $T_{\mathrm{FL}}$ is highest for the samples with the largest residual resistivity ratio (above $\sim 16$ ) (see Figs. S1(c) and S6 in the SM [27]).

A related study of $\mathrm{FeSe}_{1-x} \mathrm{~S}_{x}$ detected linear resistivity from the $35 \mathrm{~T}$ temperature dependence of the longitudinal magnetoresistance in Ref. [24], assumed to occur near the nematic critical point. In this study, the sulfur-doping level is given as the nominal concentration, which is an overestimate of $x$. For example, the $x_{\text {nom }} \sim 0.16$ suggested to be at the nematic critical point shows a structural transition at $T_{s} \sim$ $51 \mathrm{~K}$, which corresponds to $x \sim 0.13$ in our phase diagrams in Fig. 3 and in Fig. S1(b) in the SM [27] (see, also, the resistivity derivative in Ref. [24]). However, the linear resistivity in Ref. [24] agrees with our findings inside the nematic state, once corrected for the doping shift [Fig. 3(b)].

Theoretical models suggest that the temperature exponent $\gamma$ in the vicinity of nematic critical points is highly dependent on the presence of cold spots on different Fermi surfaces, due 

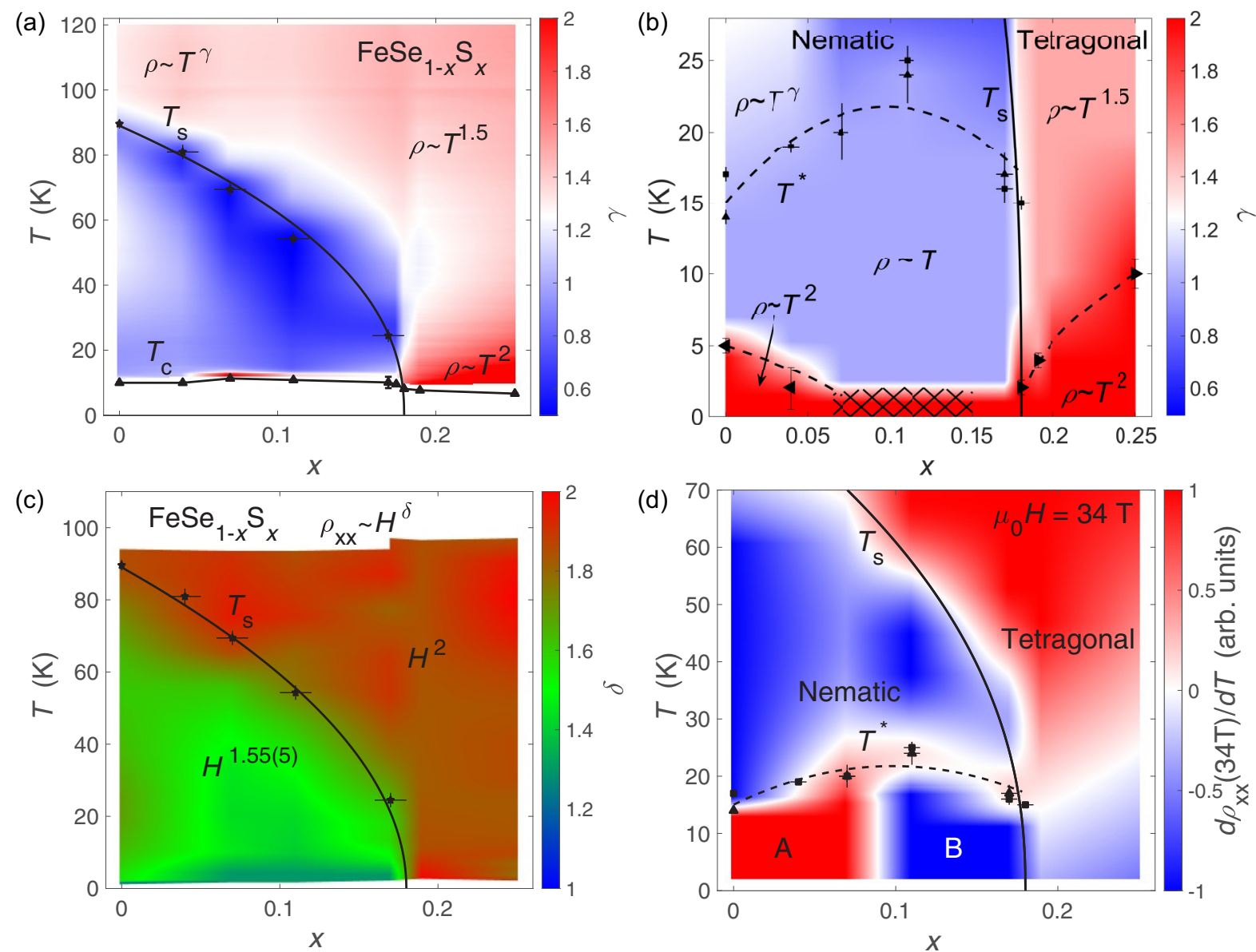

FIG. 3. Phase diagrams of the resistivity exponents and high-field transport in $\mathrm{FeSe}_{1-x} \mathrm{~S}_{x}$. The color plot of the temperature exponent $\gamma$, extracted from (a) zero-resistivity data, as shown in Fig. S10 in the SM [27]. (b) The low-temperature resistivity exponent below $T^{*}$, extrapolated from high-magnetic fields as shown in Figs. S5 and S7 in the SM [27], indicating the unusual transport behavior of the nematic phase. Fermi liquid recovers below $T_{\mathrm{FL}}$ for the compositions $x$ with lowest disorder both inside the nematic phase and in the tetragonal phase. (c) The temperature dependence of field exponent $\delta$ showing a dominant $\sim H^{1.55}$ power law inside the nematic phase (based on Fig. S3 in the SM [27] for 5-35 T). (d) The color plot of the slope of resistivity in $34 \mathrm{~T}$ between the nematic A and B phases. Solid squares represent $T_{s}$ and solid triangles represent $T_{c}$. $T^{*}$ indicated by stars represents the peak in magnetoresistance and the maximum in $\left|R_{H}\right|$. Solid lines indicate the nematic and superconducting phase boundaries and the dashed lines are guides to the eye. The hashed region at low temperatures in (b) has not yet been accessed experimentally in the longitudinal resistance experiments shown in Fig. S5 in the SM [27]. However, the samples show clear quantum oscillations in the transverse magnetoresistance, as shown in Fig. 1(c) as well as in Ref. [3], consistent with the presence of the Fermi-liquid behavior at low temperatures for all samples.

to the symmetry of the nematic order parameter $[36,39,40]$. Furthermore, resistivity near a nematic critical point can have a variation of $\gamma$ with temperature due to the scattering from acoustic phonons [22]. This is a potential cause for the variation of $\gamma$, as we observe experimentally in $\mathrm{FeSe}_{1-x} \mathrm{~S}_{x}$ near the nematic end point. Furthermore, the scale at which the crossover to Fermi-liquid behavior occurs at $T_{\mathrm{FL}}$ also depends on the strength of the coupling to the lattice [21]. To assess the critical behavior in $\mathrm{FeSe}_{1-x} \mathrm{~S}_{x}$, it is worth emphasizing that the effective masses associated to the outer hole bands do not show any divergence close to the nematic end point $x \sim 0.18$ [3]. This agrees with the variation of the $A^{1 / 2}$ coefficient (see Fig. S11 in the SM [27]) and previous studies under pressure [20], suggesting that the critical nematic fluctuations are quenched by the coupling to the lattice along certain directions in $\mathrm{FeSe}_{1-x} \mathrm{~S}_{x}$. This effect would lead to the resistivity exponent varying with temperature, as we find experimentally and predicted theoretically [22].

An overall representation of the resistivity slope in high fields $d \rho_{x x}(34 \mathrm{~T}) / d T$ for $\mathrm{FeSe}_{1-x} \mathrm{~S}_{x}$ as a function of temperature is shown in the phase diagram in Fig. 3(d). The low-temperature manifestation of the nematic $\mathrm{A}$ and $\mathrm{B}$ phases is clearly different below $T^{*}$. In order to identify possible sources of scattering responsible for these changes, we consider the role of spin fluctuations. Recent NMR data found that antiferromagnetic spin fluctuations are present inside the nematic phase of $\mathrm{FeSe}_{1-x} \mathrm{~S}_{x}$, being strongest around $x \sim$ 0.1 [26]. In FeSe, spin fluctuations are rather anisotropic [26,41] and strongly field dependent below $15 \mathrm{~K}$ [11]. Interestingly, the spin-fluctuation relaxation rate is enhanced below $T^{*}$ [Fig. 3(d)], suggesting a correlation between spindependent scattering, the high-field magnetoresistance, and 
the low-temperature transport inside the nematic state. Highmagnetic fields are expected to align magnetic spins and could affect the energy dispersion of low-energy spin excitations and spin-dependent scattering in magnetic fields. In FeSe, the spin-relaxation rate in different magnetic fields up to $19 \mathrm{~T}$ deviates at $T^{*}$ [11], but it remains relatively constant in $19 \mathrm{~T}$ at the lowest temperatures. This may suggest that the variation in magnetoresistance in high-magnetic fields at low temperatures in $\mathrm{FeSe}_{1-x} \mathrm{~S}_{x}$ is more sensitive to the changes in the electronic behavior, rather than to the spin fluctuations across the nematic phase.

In the low-temperature regime of $\mathrm{FeSe}_{1-x} \mathrm{~S}_{x}$, below $T^{*}$ we find a temperature regime with a linear resistivity across the whole nematic phase. Linear resistivity is usually found near a antiferromagnetic critical point, such as in $\mathrm{BaFe}_{2}\left(\mathrm{As}_{1-x} \mathrm{P}_{x}\right)$ [37], and this behavior is a potential manifestation of scattering induced by critical spin fluctuations in clean systems [42]. $\mu$ SR studies place FeSe near an itinerant antiferromagnetic quantum critical point at very low temperatures [12]. Spin fluctuations are present inside the nematic state in $\mathrm{FeSe}_{1-x} \mathrm{~S}_{x}$ $[11,26]$, being suppressed at the nematic end point where a Lifshitz transition was detected in quantum oscillations [3]. Thus, in $\mathrm{FeSe}_{1-x} \mathrm{~S}_{x}$, we find a qualitative link between the linear resistivity and spin fluctuations below $T^{*}$ only inside the nematic phase.

The striking difference in magnetotransport behavior between the nematic and tetragonal phases in $\mathrm{FeSe}_{1-x} \mathrm{~S}_{x}$ can have significant implications on what kind of superconductivity is stabilized inside and outside the nematic phase as different pairing channels may be dominant in different regions, as found experimentally $[18,19]$. Linear resistivity found at low temperatures inside the nematic state is present in the region where spin fluctuations exist. Furthermore, the absence of superconductivity enhancement at the nematic end point in $\mathrm{FeSe}_{1-x} \mathrm{~S}_{x}$ is supported by the lack of divergent critical fluctuations, found both with chemical pressure [3] and applied pressure [20]. It is expected that the coupling to the relevant lattice strain restricts criticality in nematic systems only to certain high-symmetry directions $[21,43]$. Future theoretical work needs to be dedicated to understanding multiband transport phenomena of $\mathrm{FeSe}_{1-x} \mathrm{~S}_{x}$ and address the role played by both small and large pockets in relation to the BEC-BCS crossover, the effect on the Zeeman energy on different bands, as well as the possible field-induced effects in scattering.

In conclusion, we have studied the evolution of the lowtemperature magnetotransport behavior in $\mathrm{FeSe}_{1-x} \mathrm{~S}_{x}$ in high- magnetic fields up to $69 \mathrm{~T}$. We find that the nematic state displays unconventional power laws in magnetic field, reflecting the dominant anomalous scattering inside the nematic phase. The temperature variation of the resistivity exponent near the nematic end point reflects the nematic elastic coupling with the lattice that also suppresses the divergent nematic critical fluctuations in $\mathrm{FeSe}_{1-x} \mathrm{~S}_{x}$. In high-magnetic fields, well above the upper critical fields, the transverse magnetoresistance shows a change in slope that reflects the changes in the spin fluctuations and/or the electronic structure. In the low-temperature limit, we find an extended linear resistivity in temperature where spin fluctuations are present. Fermi-liquid behavior recovers at low temperatures depending on the composition, impurity level, and strength of the nematoelastic coupling. Our study reveals an anomalous transport behavior of the nematic state, strikingly different from the tetragonal phase, that is likely to influence superconductivity in $\mathrm{FeSe}_{1-x} \mathrm{~S}_{x}$.

In accordance with the EPSRC policy framework on research data, access to the data will be made available from [44].

We thank Lara Befatto, Dmitrii Maslov, Rafael Fernandes, Erez Berg, Shigeru Kasahara, Steve Simon, Siddharth Parameswaran, and Stephen Blundell for useful comments and discussions. We thank and acknowledge previous contributions from Matthew Watson, Mara Bruma, Samuel Blake, Abhinav Naga, and Nathaniel Davies. This work was mainly supported by EPSRC (Grants No. EP/L001772/1, No EP/I004475/1, and No. EP/I017836/1). A.A.H. acknowledges the financial support of the Oxford Quantum Materials Platform Grant (Grant No. EP/M020517/1). A portion of this work was performed at the National High Magnetic Field Laboratory, which is supported by National Science Foundation Cooperative Agreement No. DMR-1157490 and the State of Florida. Part of this work was supported by HFML-RU/FOM and LNCMI-CNRS, members of the European Magnetic Field Laboratory (EMFL) and by EPSRC (UK) via its membership to the EMFL (Grant No. EP/N01085X/1). Part of this work was supported by Programme Investissements d'Avenir under the programme ANR-11-IDEX-0002-02, Reference No. ANR-10-LABX-0037-NEXT. We also acknowledge the Oxford Centre for Applied Superconductivity and the Oxford John Fell Fund for financial support. A.I.C. acknowledges financial support through an EPSRC Career Acceleration Fellowship (Grant No. EP/I004475/1).
[1] B. J. Ramshaw, S. E. Sebastian, R. D. McDonald, James Day, B. S. Tan, Z. Zhu, J. B. Betts, Ruixing Liang, D. A. Bonn, W. N. Hardy, and N. Harrison, Quasiparticle mass enhancement approaching optimal doping in a high-Tc superconductor, Science 348, 317 (2015).

[2] G. S. Boebinger, Yoichi Ando, A. Passner, T. Kimura, M. Okuya, J. Shimoyama, K. Kishio, K. Tamasaku, N. Ichikawa, and S. Uchida, Insulator-to-Metal Crossover in the Normal State of $\mathrm{La}_{2-x} \mathrm{Sr}_{x} \mathrm{CuO}_{4}$ Near Optimum Doping, Phys. Rev. Lett. 77, 5417 (1996).
[3] A. I. Coldea, S. F. Blake, S. Kasahara, A. A. Haghighirad, M. D. Watson, W. Knafo, E. S. Choi, A. McCollam, P. Reiss, T. Yamashita, M. Bruma, S. Speller, Y. Matsuda, T. Wolf, T. Shibauchi, and A. J. Schofield, Evolution of the low-temperature Fermi surface of superconducting $\mathrm{FeSe}_{1-x} \mathrm{~S}_{x}$ across a nematic phase transition, npj Quantum Mater. 4, 2 (2019).

[4] Ian M. Hayes, Ross D. McDonald, Nicholas P. Breznay, Toni Helm, Philip J. W. Moll, Mark Wartenbe, Arkady Shekhter, and James G. Analytis, Scaling between magnetic field and 
temperature in the high-temperature superconductor $\mathrm{BaFe}_{2}\left(\mathrm{As}_{1-x} \mathrm{P}_{x}\right)_{2}$, Nat. Phys. 12, 916 (2016).

[5] P. Giraldo-Gallo, J. A. Galvis, Z. Stegen, K. A. Modic, F. F. Balakirev, J. B. Betts, X. Lian, C. Moir, S. C. Riggs, J. Wu, A. T. Bollinger, X. He, I. Božović, B. J. Ramshaw, R. D. McDonald, G. S. Boebinger, and A. Shekhter, Scale-invariant magnetoresistance in a cuprate superconductor, Science 361, 479 (2018)

[6] A. I. Coldea and M. D. Watson, The key ingredients of the electronic structure of FeSe, Annu. Rev. Cond. Mater. Phys. 9, 125 (2018).

[7] M. D. Watson, T. K. Kim, A. A. Haghighirad, N. R. Davies, A. McCollam, A. Narayanan, S. F. Blake, Y. L. Chen, S. Ghannadzadeh, A. J. Schofield, M. Hoesch, C. Meingast, T. Wolf, and A. I. Coldea, Emergence of the nematic electronic state in FeSe, Phys. Rev. B 91, 155106 (2015).

[8] Matthew D. Watson, Steffen Backes, Amir A. Haghighirad, Moritz Hoesch, Timur K. Kim, Amalia I. Coldea, and Roser Valentí, Formation of Hubbard-like bands as a fingerprint of strong electron-electron interactions in FeSe, Phys. Rev. B 95, 081106(R) (2017).

[9] Q. Wang, Y. Shen, B. Pan, X. Zhang, K. Ikeuchi, K. Iida, A. D. Christianson, H. C. Walker, D. T .Adroja, M. Abdel-Hafiez, X. Chen, D. A. Chareev, A. N. Vasiliev, and J. Zhao, Magnetic ground state of FeSe, Nat. Commun. 7, 12182 (2016).

[10] S. Kasahara, T. Yamashita, A. Shi, R. Kobayashi, Y. Shimoyama, T. Watashige, K. Ishida, T. Terashima, T. Wolf, F. Hardy, C. Meingast, H. v. Löhneysen, A. Levchenko, T. Shibauchi, and Y. Matsuda, Giant superconducting fluctuations in the compensated semimetal FeSe at the BCS-BEC crossover, Nat. Commun. 7, 12843 (2016).

[11] Anlu Shi, Takeshi Arai, Shunsaku Kitagawa, Takayoshi Yamanaka, Kenji Ishida, Anna E. Böhmer, Christoph Meingast, Thomas Wolf, Michihiro Hirata, and Takahiko Sasaki, Pseudogap Behavior of the Nuclear Spin-Lattice Relaxation Rate in FeSe Probed by 77Se-NMR, J. Phys. Soc. Jpn. 87, 013704 (2018).

[12] V. Grinenko, R. Sarkar, P. Materne, S. Kamusella, A. Yamamshita, Y. Takano, Y. Sun, T. Tamegai, D. V. Efremov, S.-L. Drechsler, J.-C. Orain, T. Goko, R. Scheuermann, H. Luetkens, and H.-H. Klauss, Low-temperature breakdown of antiferromagnetic quantum critical behavior in FeSe, Phys. Rev. B 97, 201102(R) (2018).

[13] P. O. Sprau, A. Kostin, A. Kreisel, A. E. Böhmer, V. Taufour, P. C. Canfield, S. Mukherjee, P. J. Hirschfeld, B. M. Andersen, and J. C. Séamus Davis, Discovery of Orbital-Selective Cooper Pairing in FeSe, Science 357, 75 (2016).

[14] Shigeru Kasahara, Tatsuya Watashige, Tetsuo Hanaguri, Yuhki Kohsaka, Takuya Yamashita, Yusuke Shimoyama, Yuta Mizukami, Ryota Endo, Hiroaki Ikeda, Kazushi Aoyama, Taichi Terashima, Shinya Uji, Thomas Wolf, Hilbert von Löhneysen, Takasada Shibauchi, and Yuji Matsuda, Fieldinduced superconducting phase of FeSe in the BCS-BEC crossover, Proc. Natl. Acad. Sci. USA 111, 16309 (2014).

[15] M. D. Watson, T. K. Kim, A. A. Haghighirad, S. F. Blake, N. R. Davies, M. Hoesch, T. Wolf, and A. I. Coldea, Suppression of orbital ordering by chemical pressure in $\mathrm{FeSe}_{1-x} \mathrm{~S}_{x}$, Phys. Rev. B 92, 121108(R) (2015).

[16] P. Reiss, M. D. Watson, T. K. Kim, A. A. Haghighirad, D. N. Woodruff, M. Bruma, S. J. Clarke, and A. I. Coldea,
Suppression of electronic correlations by chemical pressure from FeSe to FeS, Phys. Rev. B 96, 121103(R) (2017).

[17] S. Hosoi, K. Matsuura, K. Ishida, Hao Wang, Y. Mizukami, T. Watashige, S. Kasahara, Y. Matsuda, and T. Shibauchi, Nematic quantum critical point without magnetism in $\mathrm{FeSe}_{1-x} \mathrm{~S}_{x}$ superconductors, Proc. Natl. Acad. Sci. 113, 8139 (2016).

[18] Yuki Sato, Shigeru Kasahara, Tomoya Taniguchi, Xiangzhuo Xing, Yuichi Kasahara, Yoshifumi Tokiwa, Youichi Yamakawa, Hiroshi Kontani, Takasada Shibauchi, and Yuji Matsuda, Abrupt change of the superconducting gap structure at the nematic critical point in $\mathrm{FeSe}_{1-x} \mathrm{~S}_{x}$, Proc. Natl. Acad. Sci. USA 115, 1227 (2018).

[19] T. Hanaguri, V. Iwaya, Y. Kohsaka, T. Machida, T. Watashige, S. Kasahara, T. Shibauchi, and Y. Matsuda, Two distinct superconducting pairing states divided by the nematic end point in $\mathrm{FeSe}_{1-x} \mathrm{~S}_{x}$, Sci. Adv. 4, eaar6419 (2018).

[20] P. Reiss, D. Graf, A. A. Haghighirad, W. Knafo, L. Drigo, M. Bristow, A. J. Schofield, and A. I. Coldea, Quenched nematic criticality and two superconducting domes in an iron-based superconductor, Nat. Phys. 16, 89 (2020).

[21] I. Paul and M. Garst, Lattice Effects on Nematic Quantum Criticality in Metals, Phys. Rev. Lett. 118, 227601 (2017).

[22] V. S. de Carvalho and R. M. Fernandes, Resistivity near a nematic quantum critical point: Impact of acoustic phonons, Phys. Rev. B 100, 115103 (2019).

[23] A. E. Böhmer, F. Hardy, F. Eilers, D. Ernst, P. Adelmann, P. Schweiss, T. Wolf, and C. Meingast, Lack of coupling between superconductivity and orthorhombic distortion in stoichiometric single-crystalline FeSe, Phys. Rev. B 87, 180505(R) (2013).

[24] S. Licciardello, J. Buhot, J. Lu, J. Ayres, S. Kasahara, Y. Matsuda, T. Shibauchi, and N. E. Hussey, Electrical resistivity across a nematic quantum critical point, Nature (London) 567, 213 (2019).

[25] S. Licciardello, N. Maksimovic, J. Ayres, J. Buhot, M. Culo, B Bryant, S. Kasahara, Y. Matsuda, T. Shibauchi, V. Nagarajan, J. G. Analytis, and N. E. Hussey, Coexistence of orbital and quantum critical magnetoresistance in $\mathrm{FeSe}_{1-x} \mathrm{~S}_{x}$, Phys. Rev. Res. 1, 023011 (2019).

[26] P. Wiecki, K. Rana, A. E. Böhmer, Y. Lee, S. L. Bud'ko, P. C. Canfield, and Y. Furukawa, Persistent correlation between superconductivity and antiferromagnetic fluctuations near a nematic quantum critical point in $\mathrm{FeSe}_{1-x} \mathrm{~S}_{x}$, Phys. Rev. B 98, 020507(R) (2018).

[27] See Supplemental Material at http://link.aps.org/supplemental/ 10.1103/PhysRevResearch.2.013309 for additional data and analysis.

[28] M. Bristow, P. Reiss, A. A. Haghighirad, and A. I. Coldea (unpublished).

[29] M. D. Watson, T. Yamashita, S. Kasahara, W. Knafo, M. Nardone, J. Béard, F. Hardy, A. McCollam, A. Narayanan, S. F. Blake, T. Wolf, A. A. Haghighirad, C. Meingast, A. J. Schofield, H. v. Löhneysen, Y. Matsuda, A. I. Coldea, and T. Shibauchi, Dichotomy between the Hole and Electron Behavior in Multiband Superconductor FeSe Probed by Ultrahigh Magnetic Fields, Phys. Rev. Lett. 115, 027006 (2015).

[30] Yue Sun, Sunseng Pyon, and Tsuyoshi Tamegai, Electron carriers with possible Dirac-cone-like dispersion in $\mathrm{FeSe}_{1-x} \mathrm{~S}_{x}$ 
$(x=0$ and 0.14$)$ single crystals triggered by structural transition, Phys. Rev. B 93, 104502 (2016).

[31] L. Fanfarillo, E. Cappelluti, C. Castellani, and L. Benfatto, Unconventional Hall Effect in Pnictides from Interband Interactions, Phys. Rev. Lett. 109, 096402 (2012).

[32] A.B. Pippard, Magnetoresistance in Metals, Cambridge Studies in Low Temperature Physics (Cambridge University Press, Cambridge, 1989).

[33] Xu Du, Shan-Wen Tsai, Dmitrii L. Maslov, and Arthur F. Hebard, Metal-Insulator-Like Behavior in Semimetallic Bismuth and Graphite, Phys. Rev. Lett. 94, 166601 (2005).

[34] A. Narayanan, M. D. Watson, S. F. Blake, N. Bruyant, L. Drigo, Y. L. Chen, D. Prabhakaran, B. Yan, C. Felser, T. Kong, P. C. Canfield, and A. I. Coldea, Linear Magnetoresistance Caused by Mobility Fluctuations in $n$-Doped $\mathrm{Cd}_{3} \mathrm{As}_{2}$, Phys. Rev. Lett. 114, 117201 (2015).

[35] John Singleton, A simple transport model for the temperaturedependent linear magnetoresistance of high-temperature superconductors, arXiv:1810.01998.

[36] Xiaoyu Wang and Erez Berg, Scattering mechanisms and electrical transport near an Ising nematic quantum critical point, Phys. Rev. B 99, 235136 (2019).

[37] S. Kasahara, T. Shibauchi, K. Hashimoto, K. Ikada, S. Tonegawa, R. Okazaki, H. Shishido, H. Ikeda, H. Takeya, K. Hirata, T. Terashima, and Y. Matsuda, Evolution from
non-Fermi- to Fermi-liquid transport via isovalent doping in $\mathrm{BaFe}_{2}\left(\mathrm{As}_{1-x} \mathrm{P}_{x}\right)_{2}$ superconductors, Phys. Rev. B 81, 184519 (2010).

[38] Takahiro Urata, Yoichi Tanabe, Khuong Kim Huynh, Hidetoshi Oguro, Kazuo Watanabe, and Katsumi Tanigaki, Non-Fermi liquid behavior of electrical resistivity close to the nematic critical point in $\mathrm{Fe}_{1-x} \mathrm{Co}_{x} \mathrm{Se}$ and $\mathrm{FeSe}_{1-y} \mathrm{~S}_{y}$, arXiv:1608.01044.

[39] Luca Dell'Anna and Walter Metzner, Electrical Resistivity Near Pomeranchuk Instability in Two Dimensions, Phys. Rev. Lett. 98, 136402 (2007).

[40] Dmitrii L. Maslov, Vladimir I. Yudson, and Andrey V. Chubukov, Resistivity of a Non-Galilean-Invariant Fermi Liquid Near Pomeranchuk Quantum Criticality, Phys. Rev. Lett. 106, 106403 (2011).

[41] R. X. Cao, Jun Dong, Q. L. Wang, X. S. Ye, J. B. Zhang, Y. F. Xu, D. A. Chareev, A. N. Vasiliev, Bing Wu, Guoqing $\mathrm{Wu}$, and $\mathrm{X}$. H. Zeng, Observation of orbital ordering and origin of nematic order in FeSe, New J. Phys. 21, 103033 (2019).

[42] A. Rosch, Interplay of Disorder and Spin Fluctuations in the Resistivity Near a Quantum Critical Point, Phys. Rev. Lett. 82, 4280 (1999).

[43] D. Labat and I. Paul, Pairing instability near a lattice-influenced nematic quantum critical point, Phys. Rev. B 96, 195146 (2017).

[44] See https://doi.org/10.5287/bodleian:QmbQQ6EQR. 